\title{
Effect of Lossy Earth on Antenna Gain, Part II
}

\author{
Walter L. Curtis
}

\author{
Contribution From Aero-Space Division, The Boeing Company, Seattle, Wash.
}

(Received February 12, 1964)

The effect of lossy earth on the far field gain of antennas with finite size metal ground planes is investigated theoretically. In particular, an approximate expression is derived for the ratio of free-space antenna gain to the gain when the antenna is mounted on the earth. The solution is based on the compensation theorem and is dependent on the solution of a closely related problem by J. R. Wait.

\section{Introduction}

The free-space pattern of an antenna may be conveniently measured using models on an antenna pattern range. The operation of the antenna in the presence of the earth can then be determined using the free-space pattern and geometrical optics as long as the antenna is not too close to the earth. When the antenna is located at the surface of the earth, there is usually no simple relationship between the free-space pattern and the actual far field radiation from the antenna. However, as shown experimentally by Coe and Curtis [1964], when a large perfectly conducting ground plane is used as part of the antenna, the effect of the earth is to reduce the radiation only at low elevation angles. This paper shows a development of a theoretical expression for the ratio of the far field of an antenna located at the surface of the earth to the far field of the same antenna in free space. The antenna considered here is a short dipole over a large metal ground plane. However, the previously mentioned experimental work indicates that the results will be approximately the same for most other antennas on the same size ground plane.

\section{Theoretical Derivation}

The antenna configuration considered here is shown in figure 1. Antenna B is a short vertical electric dipole on a metal ground plane of radius $a$ in the $x-y$ plane. Antenna $A$ is a short electric dipole located above the $x$ axis at distance $R_{0}$ from $\mathrm{B}$, and is positioned perpendicular to a line between A and B. The two situations of interest are first the presence of the earth with the surface coincident with the $x-y$ plane and second the absence of the earth. By the compensation theorem [Monteath, 1951] the difference between the mutual impedances of antennas

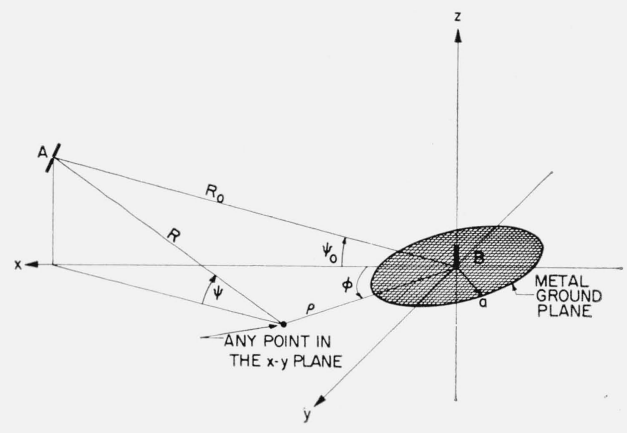

Figure 1. Location of antennas in coordinate system used to find mutual impedance. 
$\mathrm{A}$ and $\mathrm{B}$ for the two situations is given by

$$
Z_{A B}^{\prime}-Z_{A B}=\frac{1}{I_{0}^{2}} \iint_{S}\left(\bar{E}_{A} \times \bar{H}_{B}^{\prime}-\bar{E}_{B}^{\prime} \times \bar{H}_{A}\right) \cdot \overline{d s}
$$

where the terms in the expression are defined as follows: $Z_{A B}^{\prime}$ is the mutual impedance with the earth present, $Z_{A B}$ is the mutual impedance with the earth absent, $\bar{E}_{B}^{\prime}$ and $\bar{H}_{B}^{\prime}$ are the electric and magnetic fields respectively when the terminal current at $\mathrm{B}$ is $I_{0}$ with the earth present and terminal A open circuited, $\bar{E}_{A}$ and $\bar{H}_{A}$ are the electric and magnetic fields respectively when the terminal current at $\mathrm{A}$ is $I_{0}$ with the earth absent and terminal $\mathrm{B}$ open circuited, $S$, the surface of integration, is the entire $x-y$ plane.

By the nature of (1) the only components of the fields that are needed are those tangential to the $x-y$ plane. Thus assuming a perfect conducting ground plane the tangential component of both $\bar{E}_{A}$ and $\bar{E}_{B}^{\prime}$ will be zero for $\rho<a$. Equation (1) can therefore be written, with $I_{0}=1$, as

$$
Z_{A B}^{\prime}-Z_{A B}=\int_{a}^{\infty} \int_{-\pi}^{\pi}\left(\bar{E}_{A t} \times \bar{H}_{B t}^{\prime}-\bar{E}_{B t}^{\prime} \times \bar{H}_{A t}\right) \cdot \hat{z} \rho d \phi d \rho .
$$

Equation (2) is exact as written but has no practical value unless good approximations are available for most of the terms.

If we now assume that the tangential components of the electric and magnetic fields at the surface of the earth can be related by a surface impedance $(Z)$, then good approximations to all the primed quantities in (2) may be expressed as presented by Wait [1963] and Wait and Walters [1963]. Thus, after Wait,

$$
\begin{gathered}
\bar{H}_{B t}^{\prime}=\frac{i k l_{B}}{2 \pi \rho}\left(1+\frac{1}{i k \rho}\right) e^{-i k \rho} W^{\prime}(\rho, Z) \hat{\phi} \\
\bar{E}_{B t}^{\prime}=-Z H_{B t}^{\prime} \hat{\rho} \\
Z_{A B}^{\prime}=\frac{i \mu_{0} \omega l_{A} l_{B}}{2 \pi R_{0}} e^{-i k R_{0}} \cos \psi_{0} W^{\prime}\left(R_{0}, Z\right)
\end{gathered}
$$

where $l_{A}$ and $l_{B}$ are the effective lengths of antennas $\mathrm{A}$ and $\mathrm{B}$ respectively, $k=2 \pi /$ wavelength, $\mu_{0}=4 \pi \times 10^{-7}, \omega$ is the angular frequency, $\hat{\rho}$ and $\hat{\phi}$ are unit vectors, $Z$ is the surface impedance of the earth, and $W^{\prime}$ is a special function of $Z$. For $k a \gg 1 W^{\prime}$ is approximated by

$$
W^{\prime}=W(1+\Omega)
$$

where $W$ comes from the known solution of a dipole over conducting earth [Norton, 1941] and on the ground is expressed as

$$
\begin{aligned}
W(\rho, Z) & =1-i(\pi p)^{1 / 2} e^{-p} \operatorname{erfc}\left(i p^{1 / 2}\right) \\
p & =-\frac{i k \rho}{2}\left(\frac{Z}{\eta_{0}}\right)^{2} \\
\eta_{0} & =120 \pi
\end{aligned}
$$

and above ground in the far field is given as

$$
W\left(R_{0}, Z\right)=\frac{\sin \psi_{0}}{\sin \psi_{0}+\frac{Z}{\eta_{0}}}
$$


The factor $(1+\Omega)$ is a correction term tabulated by Wait and Walters [1963]. The results expressed in (6), (7), and (8) are dependent on the previously mentioned impedance boundary condition at the surface of the earth which is equivalent to the approximation that $\left|Z / \eta_{0}\right|^{2}<<1$. The remaining terms to be specified in (2) are the fields from antenna $A$ in the absence of the earth. For these we shall assume that in the region $\rho>a$ that $\bar{E}_{A t}$ and $\bar{H}_{A t}$ are the same as the fields from antenna $A$ in the absence of antenna $B$ and its metal ground plane and that $k R>>1$ (far field). Even though the limit of integration in (2) is infinite, it is reasonable to assume that the main contribution to the integral is in the region relatively close to antenna $\mathrm{B}$. If $R_{0}$ is sufficiently large, then $\bar{H}_{A t}$ can be assumed to be in the $-\hat{y}$ direction and $\bar{E}_{A t}$ in the $\hat{x}$ direction. Thus the fields from antenna $\mathrm{A}$ can be written as

$$
\begin{gathered}
\bar{H}_{A t}=-\frac{i k l_{A}}{4 \pi R} e^{-i k R \hat{y}} \\
\bar{E}_{A t}=-\eta_{0} H_{A t} \sin \psi_{0} \hat{} .
\end{gathered}
$$

Using (3), (4), (5), (9), and (10) in (2) and dividing the latter by $Z^{\prime}{ }_{A B}$ yields

$$
1-\frac{Z_{A B}}{Z_{A B}^{\prime}}=\frac{k\left[\left(Z / \eta_{0}\right)^{2}-\sin ^{2} \psi_{0}\right]\left(1+\Omega_{\psi_{0}=0}\right)}{\cos \psi_{0} \sin \psi_{0}(1+\Omega)} \int_{a}^{\infty} \int_{-\pi}^{\pi} \frac{R_{0}}{R} e^{-i k_{\rho}}\left(1+\frac{1}{i k \rho}\right) W(\rho, Z) \frac{e^{i k\left(R-R_{0}\right)}}{2 \pi i} \cos \phi d \phi d \rho .
$$

Again using the assumption that the main contribution to the integral is in the region of antenna $\mathrm{B}$, we have $R_{0} / R \approx 1$ and $k\left(R-R_{0}\right) \approx-k \rho \cos \phi \cos \psi_{0}$. Also using the integral;

$$
J_{1}(z)=\frac{1}{2 \pi i} \int_{-\pi}^{\pi} e^{i z \cos \phi} \cos \phi d \phi
$$

where $J_{1}$ is the Bessel function of the first kind of order one, (11) becomes

$$
1-\frac{Z_{A B}}{Z_{A B}^{\prime}}=\frac{k\left[\left(Z / \eta_{0}\right)^{2}-\sin ^{2} \psi_{0}\right]\left(1+\Omega_{\psi_{0}=0}\right)}{\cos \psi_{0} \sin \psi_{0}(1+\Omega)} \int_{a}^{\infty} e^{-i k_{\rho}}\left(1+\frac{1}{i k \rho}\right) W(\rho, Z) J_{1}\left(k \rho \cos \psi_{0}\right) d \rho .
$$

Since we are considering the case where $k a>>1$, we can approximate the Bessel function with the first term of its asymptotic expansion for values of $\psi_{0}$ such that $k a \cos \psi_{0}>>1$. Also approximating the factor $\left(1+\frac{1}{i k \rho}\right)$ by unity, and making the change of variable $z=\sqrt{k \rho}$ we have finally that

where

$$
\frac{Z_{A B}}{Z_{A B}^{\prime}}=1+f\left(\psi_{0}, Z\right) \int_{\sqrt{k a}}^{\infty} W(z, Z)\left[e^{-i C_{1} z^{2}}-i e^{-i C_{2} z^{2}}\right] d z
$$

$$
\begin{aligned}
f\left(\psi_{0}, Z\right) & =\frac{\left[\left(Z / \eta_{0}\right)^{2}-\sin ^{2} \psi_{0}\right]\left(1+\Omega_{\psi_{0}=0}\right) e^{-i \frac{3 \pi}{4}}}{\left(\cos \psi_{0}\right)^{3 / 2} \sin \psi_{0}(1+\Omega) \sqrt{2 \pi}} \\
W(z, Z) & =1-i(\pi p)^{1 / 2} e^{-p} \operatorname{erfc}\left(i p^{1 / 2}\right) \\
p & =-i \frac{z^{2}}{2}\left(\frac{Z}{\eta_{0}}\right)^{2} \\
C_{1} & =1-\cos \psi_{0} \\
C_{2} & =1+\cos \psi_{0} .
\end{aligned}
$$


The ratio of mutual impedance given by (14) is equal to the ratio of the far fields of antenna $\mathrm{B}$ in free space and on the ground for a constant terminal current. However, for large ground planes the change in impedance for the two situations is small and thus the square of the magnitude of (14) is approximately the change in antenna gain where

$$
\text { gain }=\frac{\text { Far field pattern intensity }}{\text { Power input to antenna } / 4 \pi}
$$

\section{Experimental Check}

The experimental work of Coe and Curtis [1964] was performed at microwave frequencies where the measured values of relative dielectric constant and loss tangent of the soil were 9 and 0.5 respectively. Thus $\left(Z / \eta_{0}\right)^{2} \approx 0.10 e^{i 26^{\circ}}$. For this particular value of soil impedance, $W(z, Z)$ may be approximated for $k a>14$ by

where $i_{1}^{2}=-3.96+i 20.5$.

$$
W(z, Z) \approx \frac{-i 10 e^{-i 26^{\circ}}}{z^{2}-z_{1}^{2}}
$$

The value of $z_{1}$ was determined by trial and error such that the function would reasonably match $W$ over the range of interest. Data presented graphically by Norton [1941] was used to determine $W$. It should be noted that for the cases considered here it is not sufficient to use the large argument asymptotic expansion of the erfc for approximating $W$. It was hoped that the simplicity of the approximate expression for $W$ would allow the integral in (14) to be expressed in terms of known tabulated functions but the author was unable to do this. The integration was, however, carried out numerically. As an aid to the numerical integration, the path of integration was shifted as shown in figure 2. Along the original path of integration $\left(C_{0}\right)$ the integrand is oscillatory and converges only as fast as $W$. Along $C_{1}$ the integrand is slowly varying and easily integrated numerically while along $C_{2}$ the integrand contains an exponential decay function which causes rapid convergence of the integral.

The resulting magnitude of (14) for several values of $k a$ is shown in figure 3 . The same curves are compared with experimental data in figure 4. The measured data shown were taken using two different antennas, a half-wavelength slot and a slot array. A complete description of the antennas and measurement technique is given in the paper by Coe and Curtis [1964].

\section{Conclusion}

A theoretical expression (14), has been derived for the ratio of free space gain to gain on the earth for a short vertical monopole antenna with a metal ground plane where $k a>>1$. The

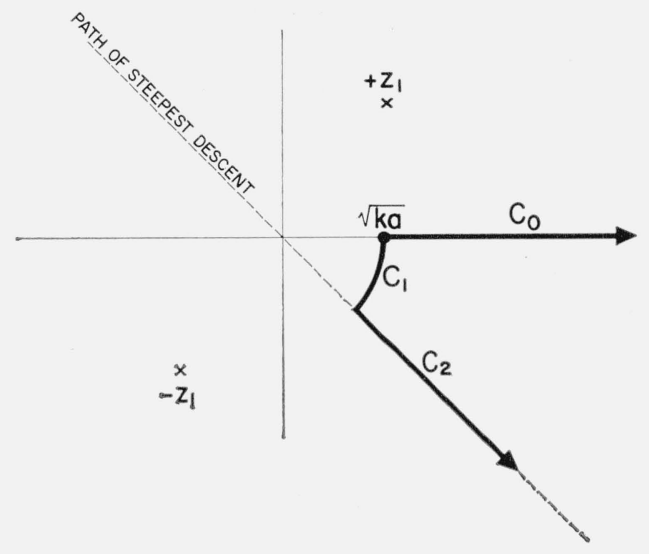

Figure 2. Complex z-plane showing change in path of integration for expediting numerical integration. 


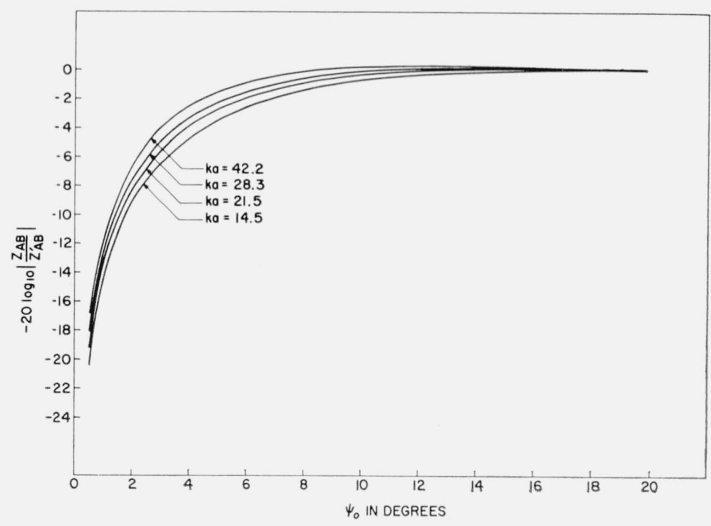

Figure 3. Plot of the magnitude of the mutual impedance ratio for several values of $k a$ and $\left(\mathrm{Z} / \eta_{0}\right)^{2}$ $\approx 0.10 \mathrm{e}^{\mathrm{i} 26^{\circ}}$.
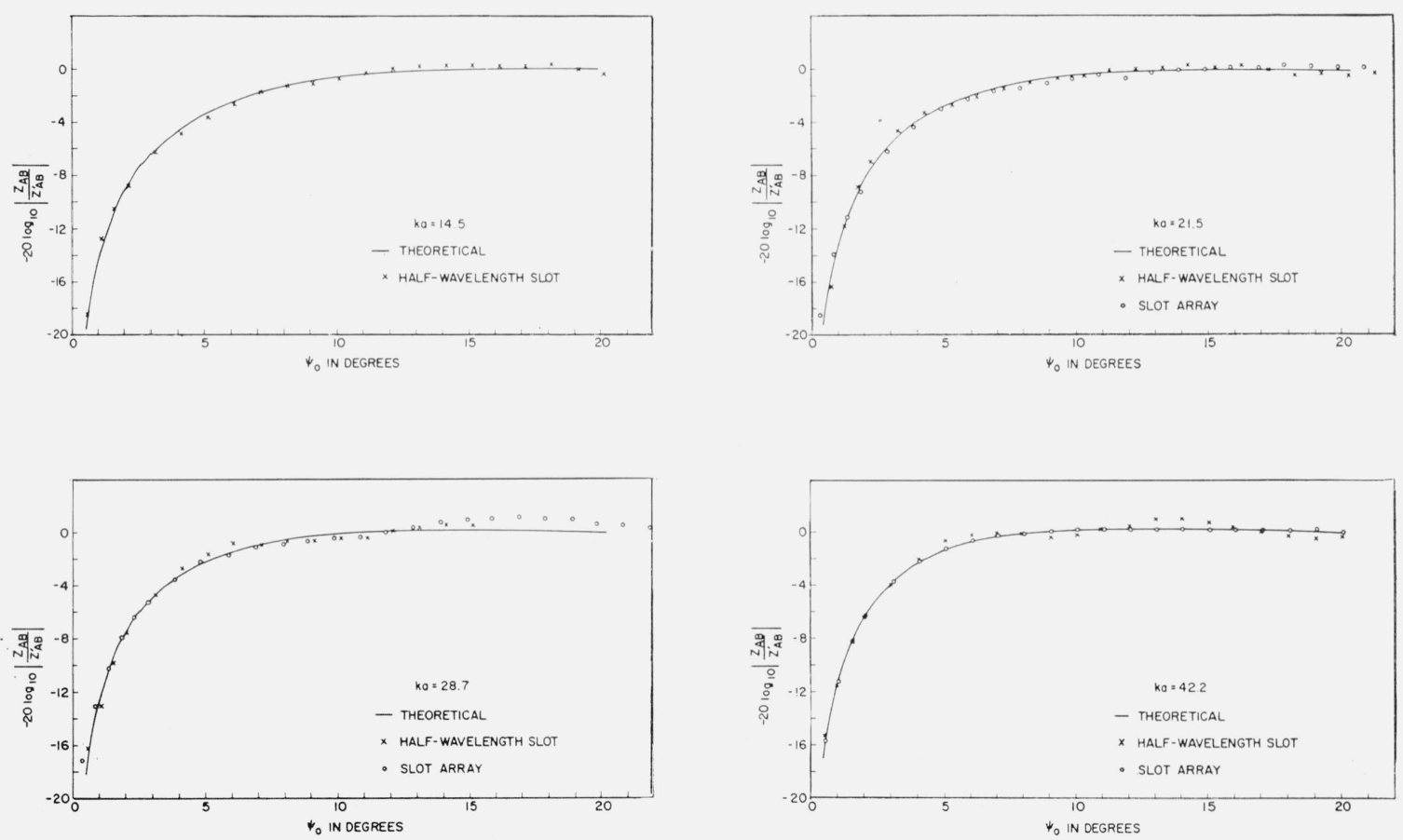

Figure 4. Comparison of theoretical change in gain with experimental data for a half-wavelength slot and a slot array.

expression is shown to agree closely with experimental data taken on different vertically polarized antennas at microwave frequencies. Based on this and the previously presented data by Coe and Curtis [1964], it is reasonable to assume that the theoretical expression will hold for all antennas whose height is small compared to the ground plane size. 


\section{References}

Coe, R. J., and W. L. Curtis (Feb. 1964), The effect of lossy earth on antenna gain, Radio Sci. J. Res. NBS/ USNC-URSI 68D, No. 2, 251-255.

Monteath, G. D. (Oct. 1951), Application of the compensation theorem to certain radiation and propagation problems, Proc. IEE 98, Part IV, 23-30.

Norton, K. A. (Dec. 1941), The calculation of ground-wave field intensity over a finitely conducting spherical earth, Proc. IRE 29, 623-639.

Wait, J. R. (1963), The theory of an antenna over an inhomogeneous ground plane, preprinted from Electromagnetic Theory and Antennas, ed. E. C. Jordon (Perganon Press, Oxford).

Wait, J. R., and L. C. Walters (1963), Influence of a sector ground screen on the field of a vertical antenna, NBS Mono. 60.

(Paper 68D7-378) 